\title{
AGaMon - A Mobile Sensor System for Breath Control
}

\author{
Rolf Seifert $^{1}$, Thorsten Conrad ${ }^{2}$, Jens Peter ${ }^{2}$, Hubert B. Keller ${ }^{1}$ \\ ${ }^{1}$ Institute of Applied Informatics (IAI), Karlsruhe Institute of Technology Hermann-von-Helmholtz-Platz \\ D-76344 Eggenstein-Leopoldshafen, Germany, \\ 2 3S GmbH, Mainzer Str. 148, D-66121 Saarbrücken \\ E-mail: rolf.seifert@kit.edu
}

\begin{abstract}
A prototype of a mobile sensor system for breath control of respiratory air is introduced which can be connected via an adapter to a smartphone for on-line monitoring. This system runs an embedded metal oxide gas sensor in a thermo-cyclic mode and determines the alcohol content on the basis of the measurement results via an innovative calibration- and evaluation procedure ProSens 3.0 in real time. The analysis results will then be displayed on the smartphone. Especially for alcohol control, the analysis demonstrates that the relative analysis error for the leading component ethanol was lower than $10 \%$ even in investigating ternary gas mixtures. Additionally, very promising results were obtained in first field tests.
\end{abstract}

Key words: mobile sensor system, breath control, thermos-cyclic operation, evaluation procedure, data analysis

\section{Introduction}

Several approaches are suggested, see e.g. [1], for applications of breath monitoring in human health care, medical applications and alcohol control. Metal oxide gas sensors (MOG) operated in thermo-cyclic mode are appropriate candidates in this context, because they are very sensitive, have a good long-term stability and are low in price. Simultaneous sampling of the conductance leads to so-called "conduction over time profiles" (CTPs) [2, 3]. These profiles can be used for component identification and concentration determination. At the Institute for Applied Computer Science of the Karlsruhe Institute of Technology (KIT), many procedures were established to evaluate such signal patterns [4] and also for source localization [5].

\section{Mobile Sensor System AGaMon}

For breath control of respiratory air, especially for alcohol control, an innovative sensor system platform was developed. Based on this platform, an adapter for smartphones was developed for mobile monitoring of the breathing air. Fig. 1 shows the pre-release prototype version of the mobile sensor system.

The sensor system runs an embedded metal oxide gas sensor in a thermo-cyclic mode and supports a variety of commercially available metal oxide gas sensors. Via its graphical user interface different parametriseable temperature cycles can be configured. Via USB, the platform is connected to a standard PC where the data live visualization and the storage is carried out. Via Bluetooth the platform can be connected to mobile applications running on smart phones.

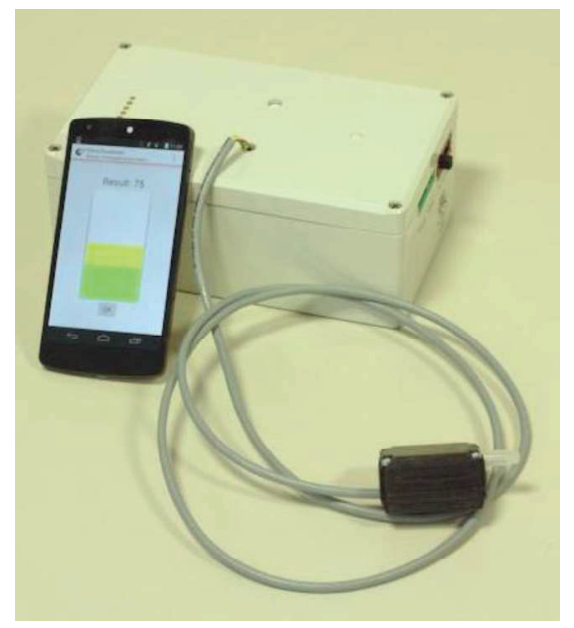

Fig. 1. Pre-release Version of the Mobile Sensor System.

An important item is that the system allows the sensors to be exposed to several interfering gases like: $\mathrm{H}_{2} \mathrm{~S}$ (which is the leading component for halitosis), $\mathrm{H}_{2}$ (leading component for dyspepsia and food intolerance), NO (leading component for asthma) or acetone (leading component for diabetes), thus covering almost all significant aspects. 


\section{Thermo-Cyclic Operation}

In the field of applications for chemical analysis of gases and volatile organic compounds (VOCs), MOGs are well introduced as gas sensing devices. This is due to the fact that they are very sensitive, have good long-term stability and are low in price. But on the other hand, when these sensor devices are operated isothermally, they are not at all selective. That means that they cannot be used for sophisticated analysis of gas mixtures. Therefore, other approaches are necessary like a gas sensor array of MOGs $[6,7]$ or by thermo cyclic operation of the MOG and simultaneous sampling of the conductance which leads to socalled "conduction over time profiles" (CTPs). These profiles give a fingerprint of the surface processes with the gas and represent the gas mixture under consideration. The gas specific features of the CTPs can be used for component identification and concentration determination. Fig. 2 shows the CTPs for acetone, ethanol and $\mathrm{H}_{2}$. It can be clearly seen that the CTPs are significant fingerprints of the three gases.

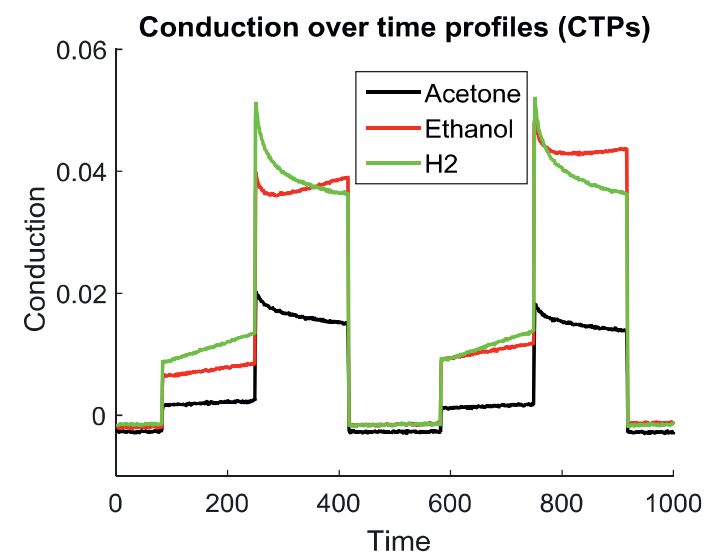

Fig. 2. Measured CTPs of acetone, athanol and $\mathrm{H}_{2}$.

For the investigations in this report, the temperature cycles in Fig. 3 were chosen, because these cycles yield the best analysis results.

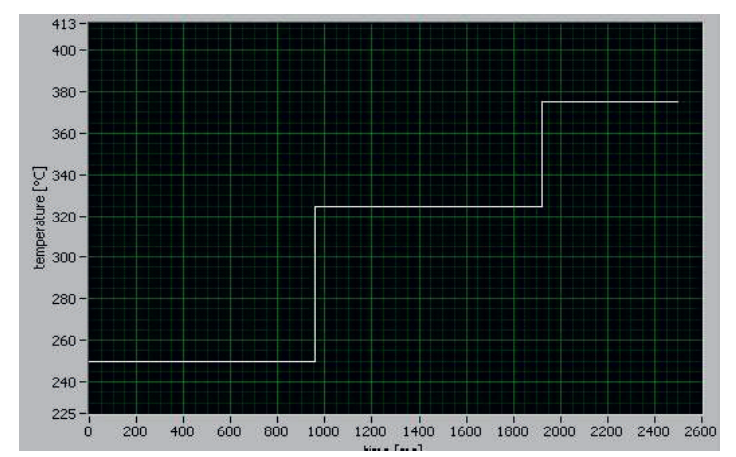

Fig. 3. Temperature cycle chosen in the following investigations.

\section{Evaluation Procedure ProSens 3.0}

For substance identification and data analysis, an innovative measurement procedure for calibration and evaluation called ProSens 3.0 is embedded in the sensor system. ProSens 3.0 is an enhancement of ProSens [8] and especially developed for investigating ternary gas mixtures.

Like ProSens, ProSens 3.0 consists of a calibration part and of an evaluation part.

In the calibration part, ProSens 3.0 determines the mathematical calibration model based on calibration measurements. The mathematical calibration model is a parametric model and only the parameters are transduced to the evaluation part.

In the evaluation part, ProSens 3.0 analysis a measured gas sample on the basis of the calibration model, performs substance identification and calculates the concentrations of the gas components.

\section{Analysis of Ternary Gas Mixtures}

For alcohol control in the respiratory air, ethanol is the leading component. But there are also other interfering gas components in the respiratory air like $\mathrm{H}_{2}$ and acetone, which may influence the measurement results. Therefore, ternary ethanol- $\mathrm{H}_{2}$-acetone gas mixtures are investigated.

For determination of the mathematical calibration model, the dosed gas samples in Tab. 1 were chosen for calibration measurements.

The investigation proved that very good substance identification could be achieved. Substance identification is very important to avoid misleading analysis results.

The determination of the concentration of the leading component ethanol was also very good (see Tab. 2). The relative analysis errors were in all considered cases smaller than $10 \%$, as can be seen in Tab. 3 . 
Tab. 1: Gas samples chosen for calibration

\begin{tabular}{|c|c|c|}
\hline $\begin{array}{c}\text { Ethanol- H2-Aceton } \\
\text { in ppm }\end{array}$ & $\begin{array}{c}\text { Ethanol- H2-Aceton } \\
\text { in ppm }\end{array}$ & $\begin{array}{c}\text { Ethanol- H2-Aceton } \\
\text { in ppm }\end{array}$ \\
\hline $50-10-0,5$ & $50-10-1,0$ & $50-10-2,0$ \\
\hline $100-10-0,5$ & $100-10-1,0$ & $100-10-2,0$ \\
\hline $175-10-0,5$ & $175-10-1,0$ & $175-10-2,0$ \\
\hline $50-20-0,5$ & $50-20-1,0$ & $50-20-2,0$ \\
\hline $100-20-0,5$ & $100-20-1,0$ & $100-20-2,0$ \\
\hline $175-20-0,5$ & $175-20-1,0$ & $175-20-2,0$ \\
\hline $50-30-0,5$ & $50-30-1,0$ & $50-30-2,0$ \\
\hline $100-30-0,5$ & $100-30-1,0$ & $100-30-2,0$ \\
\hline $175-30-0,5$ & $175-30-1,0$ & $175-30-2,0$ \\
\hline
\end{tabular}

Tab. 2: Dosed concentrations and analyzed concentration for ethanol in ppm

\begin{tabular}{|l|c|c|c|}
\hline Ethanol/H2(Acetone=0,5ppm) & 10ppm & 20ppm & 30ppm \\
\hline 50ppm & 49,6 & 49,1 & 51,2 \\
\hline 100ppm & 100,3 & 102,7 & 100,1 \\
\hline 135ppm & $\mathbf{1 3 1 , 5}$ & $\mathbf{1 4 1 , 0}$ & $\mathbf{1 3 3 , 8}$ \\
\hline 175ppm & 177,0 & 177,7 & 176,2 \\
\hline Ethanol/H2(Acetone=1,0ppm) & $\mathbf{1 0 p p m}$ & $\mathbf{2 0 p p m}$ & $\mathbf{3 0 p p m}$ \\
\hline 50ppm & 50,6 & 50,5 & 50,0 \\
\hline 100ppm & 102,1 & 99,0 & 99,8 \\
\hline 135ppm & $\mathbf{1 2 6 , 4}$ & $\mathbf{1 3 9 , 0}$ & $\mathbf{1 4 2 , 1}$ \\
\hline 175ppm & 174,7 & 174,0 & 172,7 \\
\hline Ethanol/H2(Acetone=2,0ppm) & $\mathbf{1 0 p p m}$ & $\mathbf{2 0 p p m}$ & $\mathbf{3 0 p p m}$ \\
\hline 50ppm & $\mathbf{4 9 , 7}$ & $\mathbf{4 9 , 9}$ & $\mathbf{4 9 , 0}$ \\
\hline 100ppm & 98,0 & 98,2 & 100,3 \\
\hline 135ppm & $\mathbf{1 2 8 , 0}$ & $\mathbf{1 3 6 , 2}$ & $\mathbf{1 2 3 , 5}$ \\
\hline 175ppm & 173,1 & 173,1 & 176,0 \\
\hline
\end{tabular}

Tab. 3: Relative analysis errors for the ethanol concentration determination in $\%$

\begin{tabular}{|l|c|c|c|}
\hline Ethanol/H2(Acetone=0,5ppm) & $\mathbf{1 0 p p m}$ & $\mathbf{2 0 p p m}$ & $\mathbf{3 0 p p m}$ \\
\hline 50ppm & 49,6 & 49,1 & 51,2 \\
\hline 100ppm & 100,3 & 102,7 & 100,1 \\
\hline 135ppm & $\mathbf{1 3 1 , 5}$ & $\mathbf{1 4 1 , 0}$ & $\mathbf{1 3 3 , 8}$ \\
\hline 175ppm & 177,0 & 177,7 & 176,2 \\
\hline Ethanol/H2(Acetone=1,0ppm) & $\mathbf{1 0 p p m}$ & $\mathbf{2 0 p p m}$ & $\mathbf{3 0 p p m}$ \\
\hline 50ppm & 50,6 & 50,5 & 50,0 \\
\hline 100ppm & 102,1 & 99,0 & 99,8 \\
\hline 135ppm & $\mathbf{1 2 6 , 4}$ & $\mathbf{1 3 9 , 0}$ & $\mathbf{1 4 2 , 1}$ \\
\hline 175ppm & 174,7 & 174,0 & 172,7 \\
\hline Ethanol/H2(Acetone=2,0ppm) & $\mathbf{1 0 p p m}$ & $\mathbf{2 0 p p m}$ & $\mathbf{3 0 p p m}$ \\
\hline 50ppm & 49,7 & 49,9 & 49,0 \\
\hline 100ppm & 98,0 & 98,2 & 100,3 \\
\hline 135ppm & $\mathbf{1 2 8 , 0}$ & $\mathbf{1 3 6 , 2}$ & $\mathbf{1 2 3 , 5}$ \\
\hline 175ppm & 173,1 & 173,1 & 176,0 \\
\hline
\end{tabular}




\section{Field Test}

To demonstrate the applicability of the sensor system AGaMon and ProSens3.0, a first field test was performed. In this test, several persons (probands) have drunk dosed amounts of alcohol over the time. The alcohol content was determined by a reference method and then compared with the measured results using the sensor systems. A strong correlation between the two measurement results could be recognized (see Fig. 4).

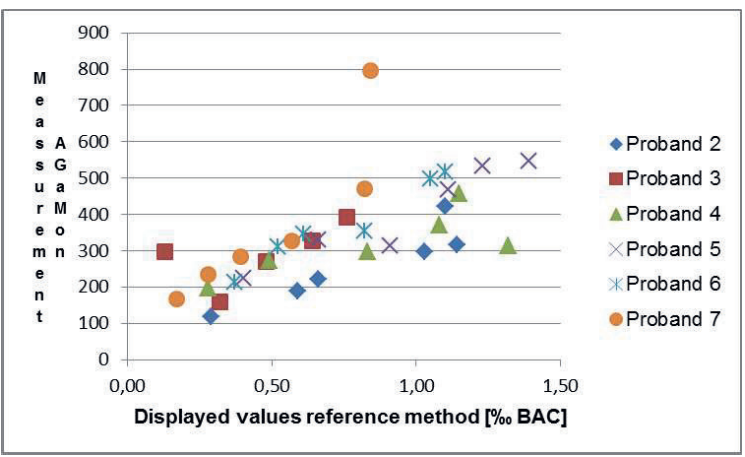

Fig. 4. Comparison of the measurement results by AGaMon with the reference values.

This is a very promising result for the further advancement and justification of the sensor system.

\section{Conclusions}

In this report, an innovative mobile sensor system called AGaMon for breath control is introduced. The sensor system runs an embedded metal oxide gas sensor in a thermocyclic mode. This operation mode enables substance identification and concentration determination as well. Especially for alcohol control in the breathing air, ethanol is the leading component. But there are also other interfering gases like $\mathrm{H}_{2}$ and acetone which may influence the measurement results.

Therefore, ternary ethanol- $\mathrm{H}_{2}$-acetone gas mixtures were investigated. The analysis with the calibration and evaluation procedure ProSens 3.0 yielded very good results. In all considered cases, the analysis errors for the concentration determination of the leading component ethanol were smaller than $10 \%$.

A first field test showed also very promising results. A strong correlation between the results obtained by the AGaMon measurements and the results obtained by reference measurements could be recognized. But nevertheless, further investigations have to be performed to improve the performance of the system and to identify the reasons of the outliers in the field test.

\section{Acknowledgements}

The research and development project was supported by the Central Innovation Program for small and medium-sized enterprises (SME) of the German Bundesministerium für Wirtschaft und Technologie (BMWi).

\section{References}

[1] K.-H. Kim, , Shamin Ara Jahan, Ehsanul Kabir, TrAC Trends in Analytical Chemistry 33, 18(2012).; doi: 10.1016/j.trac.2011.09.013

[2] A. Jerger, H. Kohler, F. Becker, H. B. Keller, R. Seifert, New applications of tin oxide gas sensors II. Intelligent sensor system for reliable monitoring of ammonia leakages, Sensors and Actuators B: Chemical, 301-307(2002); doi: 10.1016/S09254005(01)00970-4

[3] K. Frank, V. Magapu, V. Schindler, H. Kohler, H. B. Keller, R. Seifert, Chemical Analysis with Tin Oxide Gas Sensors: Choice of Additives, Method of Operation and Analysis of Numerical SignalSensors Letters 6, 908-911(2008); doi: $10.1166 /$ sl.2008.527

[4] R. Seifert, H. Keller, and J. Matthes, Innovative Procedures to Analyze Data of Gas Sensor Systems and Gas Sensor Nets: a Review, Sensors \& Transducers Journal, Vol. 184, Issue 1, 1-10(2015)

[5] J. Matthes, L. Gröll, and H. B. Keller, Source localization based on pointwise concentration measurementsSensors and Actuators A: Physical, Volume 115, Issue 1, 32-37(2004); doi:10.1016/j.sna.2004.03.016.

[6] P. Althainz, J. Goschnick, S. Ehrmann, and H. J. Ache, Sens. Actuators, B33, 72(1996;) doi:10.1016/0925-4005(96)01838-2

[7] V. V. Sysoev, I. Kiselev, M. Frietsch, and J. Goschnick, Sensors 4, 37 (2004); doi:10.3390/s40400037

[8] R. Seifert, H. B. Keller, K. Frank, H. Kohler, ProSens-An Efficient Mathematical Procedure for Calibration and Evaluation of Tin Oxide Gas Sensor DataSensor Letters, Vol. 9/1, 7-10 (2011); doi: $10.1166 /$ sl.2011.1408 